\title{
The effect of topical treatments for CRS on the sinonasal epithelial barrier*
}

\author{
Mahnaz Ramezanpour ${ }^{1}$, Aaron Rayan', Jason L.P. Smith², Sarah Vreugde' \\ Department of Surgery - Otorhinolaryngology Head and Neck Surgery, the Queen Elizabeth Hospital, and the University of \\ Adelaide, Adelaide, South Australia \\ School of Biology, Faculty of Science and Engineering, Flinders University of South Australia, Adelaide, South Australia
}

Rhinology 55: 161-169, 2017

https://doi.org/10.4193/Rhino16.284

*Received for publication:

September 30, 2016

Accepted: January 19, 2017

\begin{abstract}
Background: Several topical treatments are used in the management of Chronic Rhinosinusitis (CRS), some of which the safety and efficacy has yet to be determined. The purpose of this study was to investigate the effect of commonly used topical treatments on the sinonasal epithelial barrier.
\end{abstract}

Methods: Normal saline ( $0.9 \%$ Sodium Chloride), hypertonic saline ( $3 \%$ Sodium Chloride), FESS Sinu-Cleanse Hypertonic, FLO Sinus Care and Budesonide $1 \mathrm{mg} / 2 \mathrm{ml}$ were applied to the apical side of air-liquid interface (ALI) cultures of primary human nasal epithelial cells (HNECs) from CRS patients $(n=3)$ and non-CRS controls $(n=3)$ for 24 hours. Epithelial barrier structure and function was assessed using trans-epithelial electrical resistance (TEER), measuring the passage of Fluorescein Isothiocyanate labelled Dextrans (FITC-Dextrans) and assessing the expression of the tight junction protein Zona Occludens-1 (ZO-1) using immunofluorescence. Toxicity was assessed using a Lactate Dehydrogenase $(\mathrm{LDH})$ assay. Data was analysed using ANOVA, followed by Tukey HSD post hoc test.

Results: Hypertonic solution and budesonide significantly increased TEER values in CRS derived HNECs. In contrast, FESS SinuCleanse Hypertonic significantly reduced TEER 5 minutes after application of the solution followed by an increase in paracellular permeability of FITC-Dextrans (30 minutes) and increased LDH levels 6 hours after application of the solution.

Conclusions: Our findings confirm that isotonic and hypertonic saline solutions do not compromise epithelial barrier function in vitro but underscore the importance of examining safety and efficacy of over-the-counter wash solutions.

Key words: chronic rhinosinusitis, intranasal medication, human nasal epithelial cells, air-liquid interface, mucosal barrier

\section{Introduction}

Chronic rhinosinusitis (CRS) is a significant health problem with unclear pathophysiology. It is one of the most prevalent chronic illnesses worldwide and affects approximately $10.5 \%$ of western populations ${ }^{(1)}$. Breakdown of the mucosal barrier has been shown to contribute to tissue damage and inflammation in the paranasal sinuses (2). Relatively few studies have evaluated the epithelial barrier in CRS ${ }^{(3,4)}$. Mucosal barrier dysfunction is frequently associated with alteration of the tight junction (TJ) composition and structure. The integrity of tight junctions of the sinonasal epithelium can be affected by Staphylococcus aureus infection and secreted toxins ${ }^{(5,6)}$, Th17 cytokine families ${ }^{(7)}$ as well as in patients affected by immunodeficiency ${ }^{(8,9)}$. Despite the high prevalence and a large financial burden of CRS on health care systems, there are no Food and Drug Administration-approved drugs for the management and treatment of CRS $(10,11)$. This is due to the lack of research into safe and effective treatments, which in turn is related to the poorly understood pathophysiological mechanisms of the disease. Medical treatments for CRS include antibiotics and corticosteroids ${ }^{(12,13)}$. However, lack of clinical evidence for the efficacy of antibiotics for the treatment of CRS, makes this therapy controversial ${ }^{(14)}$. The safety 
profiles of Intranasal Corticosteroids (INCs) have been well established over 30 years of use. The systemic bioavailability of INCs such as fluticasone propionate, beclomethasone and budesonide varies from $1 \%$ to around $30 \%$ and is much lower than oral agents such as dexamethasone with systemic bioavailability of $76 \%$, thereby minimizing the risk of systemic adverse events (15). Clinical evidence demonstrates the efficacy of INCs for the management of allergic rhinitis, acute and chronic rhinosinusitis and nasal polyps ${ }^{(16)}$. Studies have also shown that irrigation with hypertonic saline was efficacious and resulted in improvement in the symptoms of CRS in over $70 \%$ of subjects ${ }^{(17,18)}$. However, with the myriad of current topical treatments used in CRS, it remains a priority to study the safety profile of the intranasal agents and their effect on the sinonasal mucosal barrier. This study investigates the effect of current topical treatments for CRS on the epithelial barrier structure and function.

\section{Materials and methods}

Human primary nasal epithelial cells

Ethical approval for nasal brushings from CRS patients and healthy volunteers was granted from The Queen Elizabeth Hospital Human Ethics Committee and only consented patients were included in the study. Human Nasal Epithelial Cells (HNECs) were isolated from patients that do not have evidence of CRS (control) and from patients with CRS without nasal polyps (CRSsNP) at the time of endoscopic sinus surgery. HNECs were harvested from paranasal sinuses by gentle brushing as described by Malik et al. ${ }^{(6)}$. Extracted cells were suspended in Bronchial Epithelial Growth Media (BEGM, CC-3170, Lonza, Walkersvill, MD, USA), supplemented with $2 \%$ Ultroser G (Pall Corporation, Port Washington, NY, USA). The cell suspension was depleted of monocytes using anti-CD68 (Dako, Glostrup, Denmark) coated culture dishes, and HNECs expanded in routine cell culture conditions of $37^{\circ} \mathrm{C}$ humidified air with 5\% CO2 in collagen coated flasks (Thermo Scientific, Walthman, MA, USA). HNECs were tested at passage two, and confirmed to be of epithelial lineage via reactivity to pan-Cytokeratin (+) and CD45 (-) antibodies (both Abcam, Cambridge, MA, USA).

\section{Air Liquid Interface Culture}

HNECs were maintained at an Air Liquid Interface (ALI) medium, following the Lonza ALI culture method (Lonza, Walkersville, USA) as described previously ${ }^{(7)}$. Transwells permeable inserts with $0.4 \mu \mathrm{m}$ pores (BD Biosciences, San Jose, CA, USA) were coated with collagen (Stemcell Technologies, Australia). 70,000 cells were seeded into the apical chamber in $100 \mu \mathrm{l}$ B-ALI growth medium and $500 \mu \mathrm{l}$ of B-ALI growth medium was added to the basal chamber. In the airlift step, B-ALI growth medium was removed from the apical and basal chambers and $500 \mu \mathrm{B}$ B-ALI differentiation medium was added to the basal chamber. The cultures were fed every second day by adding B-ALI complete differentiation medium to the basal chamber.

\section{Intranasal medications}

Normal saline (0.9\% Sodium Chloride, Baxter, Australia), hypertonic saline (3\% Sodium Chloride, Baxter, Australia), Isotonic solution (Sodium Chloride, Potassium Chloride, Sodium Bicarbonate, Glucose Anhydrous and Calcium Lactate Pentahydrate) (FLO Sinus Care, ENT Technologies, Australia), Fess Sinu-Cleanse Hypertonic (Sodium Chloride, Sodium Bicarbonate and Xylitol) (Care Pharmaceuticals, Australia) and Budesonide inhalation suspension $1 \mathrm{mg} / 2 \mathrm{ml}$ (Pulmicort, Sweden) were applied in concentrations as directed by the manufacturer to the apical chamber.

\section{Epithelial barrier permeability measurements}

Trans-Epithelial Electrical Resistance (TEER) was measured with an EVOMX volt-ohm-meter (World Precision Instruments, Sarasota, FL, USA). Briefly, $100 \mu$ l of B-ALI growth medium was added to the apical chamber of ALI Cultures to form an electrical circuit across the cell monolayer and into the basal chamber. Cultures were maintained at $37^{\circ} \mathrm{C}$ during the measurement period using a heating platform. The resistance typically reached a plateau after 21 days with a value of $500 \Omega / \mathrm{cm}^{2}$, indicating that a continuous cell monolayer with junctions was fully formed. Intranasal Medications were then added to the apical chamber of each well, and TEER measurements were obtained at different time-points up to $24 \mathrm{~h}$.

\section{Permeability assay}

Paracellular permeability was studied by measuring the apicalto-basolateral flux of FITC-dextran 4 kDa (Sigma, St Louis, USA). Briefly, after treating the cells for $24 \mathrm{~h}, 3 \mathrm{mg} / \mathrm{ml}$ of FITC-dextran was added to the apical chamber. After $2 \mathrm{~h}$ incubation, $40 \mu \mathrm{l}$ samples were taken from the basolateral chamber and serially diluted on a 96 well plate (Corning ${ }^{\circledR} \operatorname{Costar}^{\circledast}$ cell culture plates, USA), and fluorescence was measured with a microplate fluorometer (FLUOstar Optima, BMG Labtech, Ortenberg, Germany).

\section{Cytotoxicity assay}

All treatments were applied to the HNEC air liquid interface cultures and incubated for $24 \mathrm{~h}$. The amount of lactate dehydrogenase (LDH) in the medium was measured with a Cytotoxicity Detection Kit (Promega, USA). Briefly, $50 \mathrm{ml}$ of the media from each well was transferred to a new plate, and $50 \mathrm{ml}$ of LDH reagent was added to the supernatant and incubated for 30 minutes in the dark at room temperature. The OD was measured at $490 \mathrm{~nm}$ on a FLUOstar OPTIMA plate reader (BMG Labtech, Ortenberg, Germany).

Immunofluorescence microscopy

Cells were fixed with $2.5 \%$ formalin in phosphate-buffered saline 
(PBS) for $10 \mathrm{~min}$, and then the cells were permeabilized with $1 \%$ SDS in PBS. Mouse monoclonal anti-human ZO-1 (Invitrogen, Carlsbad, CA, USA), diluted to $5 \mu \mathrm{g} / \mathrm{ml}$ in TBST-10\% SFB was added for 1 hour at room temperature. Excess primary antibody was removed, and $2 \mathrm{\mu g} / \mathrm{ml}$ anti-mouse Alexa-Fluor 488 conjugated secondary antibody (Jackson ImmunoResearch Labs Inc., West Grove, PA, USA) was added and incubated overnight at $4{ }^{\circ} \mathrm{C}$. The Membranes were rinsed in TBST, and after the third wash, $200 \mathrm{ng} / \mathrm{ml}$ of 4', 6-diamidino-2-phenylindole (DAPI; Sigma, Aldrich) was added to resolve nuclei. Membranes were transferred to a glass slide and a drop of anti-fade mounting medium (Dako, Glostrup, Denmark) was added before cover-slipping. Samples were visualized by using a LSM700 confocal laser scanning microscope (Zeiss Microscopy, Germany).

\section{Statistical analysis}

Data is presented as the mean \pm SEM. The TEER statistical analysis was carried out using t-tests and all other analysis was performed using ANOVA, followed by Tukey's HSD post hoc test using SPSS (version 22).

\section{Results}

Effect of topical treatments on Trans-Epithelial Electrical Resistance (TEER) of HNEC monolayers derived from controls and CRS patients.

ALI cultures were established using HNECs isolated from 6 donors; 3 CRSsNP (all males, non-smoker, aged 45-65 years, nonallergic) and 3 controls ( 2 males, 1 female, aged 30-50 years, non-allergic). Two CRS patients were diagnosed with asthma and one had Aspirin- Exacerbated Respiratory Disease (AERD). The effect of normal saline, hypertonic saline, FESS Sinu-Cleanse Hypertonic solution, FLO Sinus Care isotonic solution, and Budesonide inhalation suspension $1 \mathrm{mg} / 2 \mathrm{ml}$ on epithelial barrier function was examined by measuring the TEER and passage of FITC-Dextrans across the HNEC monolayers after $24 \mathrm{~h}$. Sodium Dodecyl Sulphate (SDS) and B-ALI complete medium was used as a positive and negative control respectively. FESS Sinu-Cleanse Hypertonic caused a significant reduction in TEER (average of 12.5 times and 5.18 times for CRSsNP and Control patients $p=0.001$ and $p=0.01$ respectively) after $24 \mathrm{~h}$ of incubation. Other formulations did not show any significant reduction in TEER, however, hypertonic saline $(p=0.035)$ and Budesonide $(p=0.008)$ significantly increased TEER in CRS patients after $24 \mathrm{~h}$ of incubation (Figure 1).

Effect of topical treatments on paracellular permeability of FITC-Dextrans of HNEC monolayers derived from controls and CRS patients.

Application of normal saline, hypertonic saline, FLO Sinus Care and Budesonide $1 \mathrm{mg} / 2 \mathrm{ml}$ did not have a significant effect on the paracellular permeability in HNEC monolayers sourced from
A
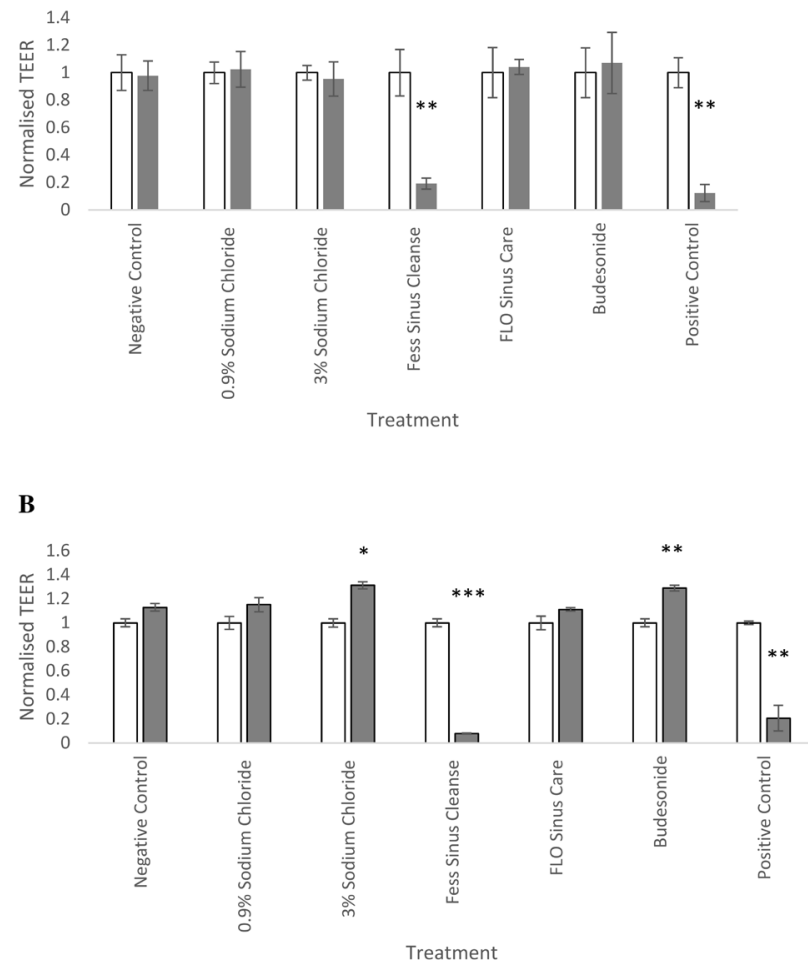

Figure 1. Effect of topical treatments on Trans-Epithelial Electrical Resistance (TEER) of HNEC monolayers derived from controls and CRS patients. TEER values, normalised to the measurement prior to treatment (white bars) and 24 hours after (grey bars) application of normal saline ( $0.9 \%$ Sodium Chloride), hypertonic saline ( $3 \%$ Sodium Chloride), FESS Sinu-Cleanse Hypertonic solution, FLO Sinus Care isotonic solution and Budesonide inhalation suspension $1 \mathrm{mg} / 2 \mathrm{ml}$ in HNEC monolayers from non-CRS control patients (A) and CRSsNP patients (B). Sodium Dodecyl Sulphate (SDS) in B-ALI complete medium was used as a positive control. The values are shown as means \pm SEM for $n=3 .^{*}=p<0.05,{ }^{* *}=p<0.01$ and ${ }^{* *}=p<0.001$, using t-tests.

CRSsNP patients and controls. FESS Sinu-Cleanse Hypertonic applied to HNECs grown at ALI caused a statistically significant increase in paracellular permeability of FITC-Dextrans after $24 \mathrm{~h}$ of incubation (average of 12 fold and 13 fold increase for CRS and Control patients respectively, $(p<0.01)$ ) (Figure 2$)$. Sodium Dodecyl Sulphate (SDS) and B-ALI complete medium was used as a positive and negative control respectively.

Effect of topical treatments on Lactate Dehydrogenase release from HNEC monolayers derived from controls and CRS patients. A standard LDH assay showed significantly reduced numbers of viable cells 24 hours after application of FESS Sinu-Cleanse Hypertonic $(p<0.01$ ) to HNEC monolayers sourced from CRSsNP patients and controls (95.9 and $94.2 \%$ reduction respectively). There was no toxicity observed after $24 \mathrm{~h}$ incubation with all other tested treatments $(p>0.05)$ (Figure 3). Sodium Dodecyl 
$\mathbf{A}$
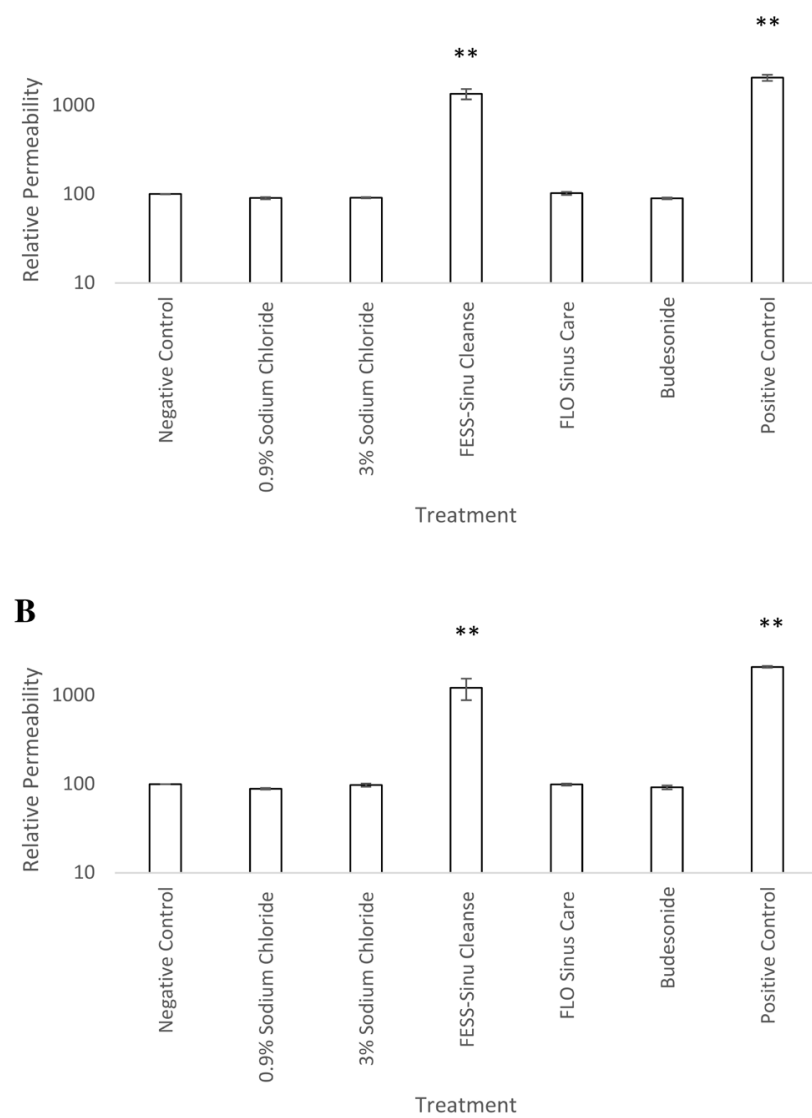

Figure 2. Paracellular permeability of HNEC monolayers derived from CRS patients and Controls. Passage of FITC-Dextrans measured 24 hours after application of normal saline ( $0.9 \%$ Sodium Chloride), hypertonic saline (3\% Sodium Chloride), FESS Sinu-Cleanse Hypertonic solution, FLO Sinus Care isotonic solution and Budesonide inhalation suspension $1 \mathrm{mg} / 2 \mathrm{ml}$ in HNEC monolayers from non-CRS control patients (A) and CRSsNP patients (B). The values are shown as means \pm SEM for $n=3$ with a logarithmic scale on the $y$-axis. Treatments significantly different from the untreated control at $\mathrm{P}<0.01$ are presented as ${ }^{* *}$. ANOVA, followed by Tukey HSD post hoc test.

Sulphate (SDS) in B-ALI complete medium was used as a positive control.

Effect of topical treatments on the immunolocalization of the tight junction protein Zona Occludens-1 (ZO-1) in HNEC monolayers from controls and CRS patients.

The effect of the different solutions on the localization of ZO-1 was examined by using immunofluorescence and confocal laser scanning microscopy, 24 hours after application of the treatments. In untreated cells, ZO-1 was located at the periphery of the apical side of the monolayer, as expected. Similarly, normal saline, hypertonic saline, FLO Sinus Care and Budesonide $1 \mathrm{mg} /$ $2 \mathrm{ml}$ led to no alterations in the localization of ZO-1. In con-
A

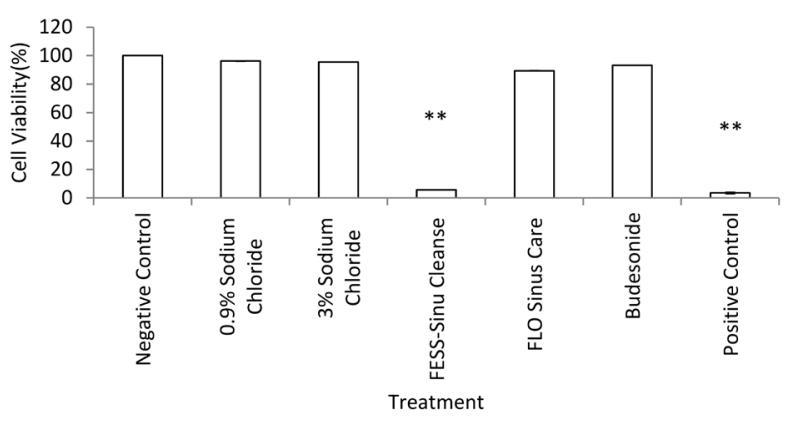

B

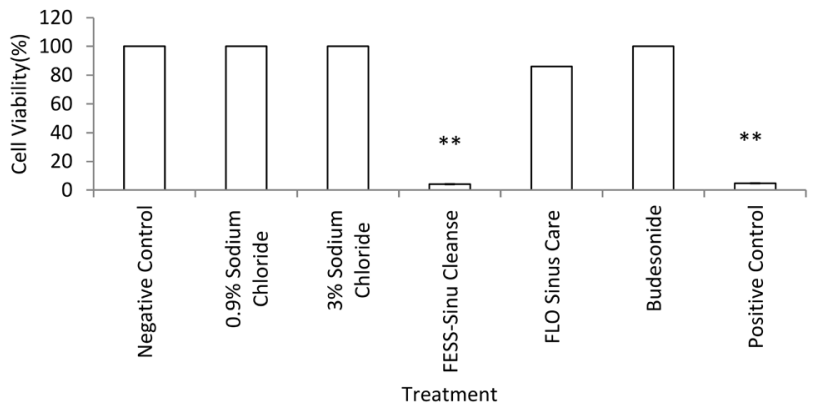

Figure 3. Cell viability of HNEC monolayers derived from CRS patients and Controls. Viability relative to no treatment control cells as determined by the LDH assay, $24 \mathrm{~h}$ after application of normal saline $(0.9 \%$ Sodium Chloride), hypertonic saline (3\% Sodium Chloride), FESS SinuCleanse Hypertonic solution, FLO Sinus Care and Budesonide inhalation suspension $1 \mathrm{mg} / 2 \mathrm{ml}$ in HNEC monolayers from non-CRS control patients (A) and CRSsNP patients (B). Cell viability was calculated relative to the untreated cells as negative control. Sodium Dodecyl Sulphate (SDS) in B-ALI complete medium was used as a positive control. The values are shown as means $\pm S E M, n=3 .^{* *}=p<0.01$. ANOVA, followed by Tukey HSD post hoc test.

trast, application of FESS Sinu-Cleanse Hypertonic resulted in profound disruption of ZO-1 immunolocalisation evidenced by faint or absent fluorescence (green staining in Figure 4). DAPI staining revealed that only few cells were remaining.

\section{Effect of topical treatments on Trans-Epithelial Electrical} Resistance (TEER) of HNEC monolayers over time.

ALI cultures were established using HNECs isolated from 3 non-CRS controls patients. All patients underwent septoplasty (3 males, aged 40-50 years) with no allergy. The effect of topical treatments on mucosal barrier function and LDH release was assessed only for those treatments that compromised barrier function 24 hours after application. FESS Sinu-Cleanse Hypertonic reduced TEER at all time-points compared to no treatment control ( $5 \mathrm{~min}, 10 \mathrm{~min}, 15 \mathrm{~min}, 20 \mathrm{~min}, 30 \mathrm{~min}, 1 \mathrm{~h}, 2 \mathrm{~h}, 4 \mathrm{~h}$ and 6 h) $(p<0.001)$. 
A

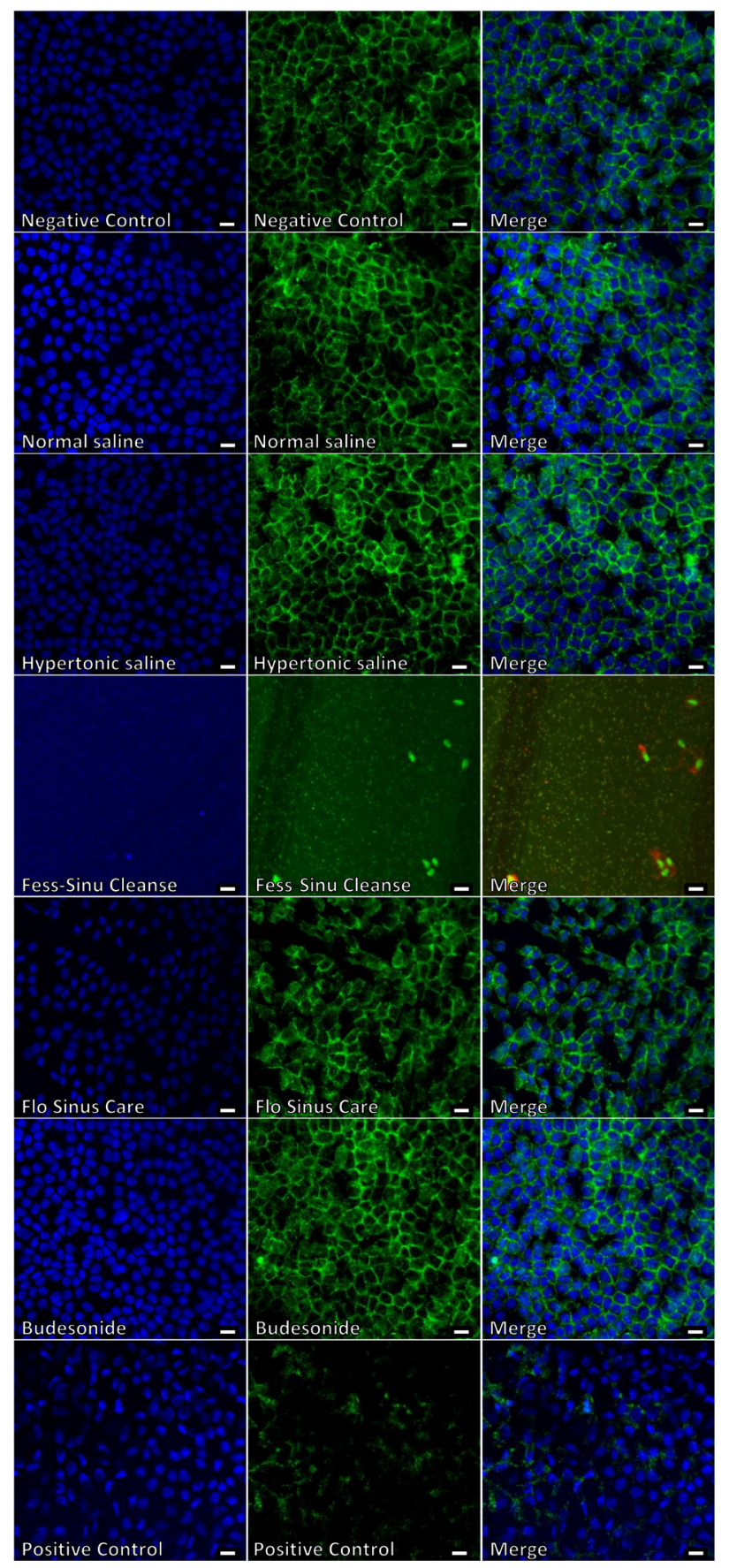

B

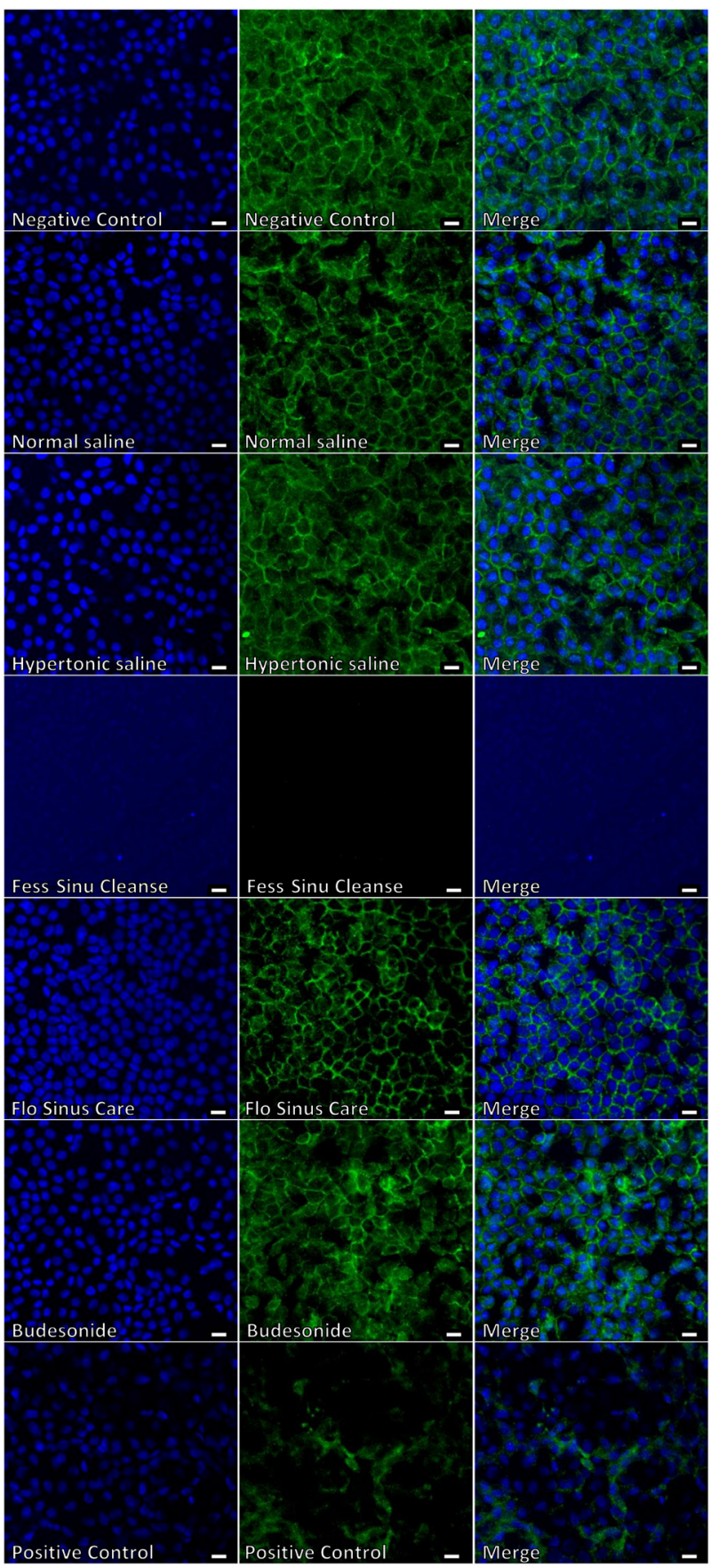

Figure 4. Immunolocalization of ZO-1 in HNEC monolayers of CRS patients and controls. Effect of normal saline (0.9\% Sodium Chloride), hypertonic saline (3\% Sodium Chloride), FESS Sinu-Cleanse Hypertonic solution, FLO Sinus Care isotonic solution and Budesonide inhalation suspension $1 \mathrm{mg} /$ $2 \mathrm{ml}$ on Zona Occludens-1 (ZO-1) immunolocalization (green staining) of HNEC monolayers of controls (A) and CRSsNP patients (B). DAPI was used to stain the nuclei (blue staining). 20X magnification, using confocal laser scanning microscopy. White bar is $20 \mu \mathrm{m}$.

Effect of topical treatments on paracellular permeability of HNEC monolayers over time.

FESS Sinu-Cleanse Hypertonic led to a significant increase in paracellular permeability of HNEC monolayers from control patients 30 minutes after application $(p<0.01)$ (Figure 6$)$.
Relative to no treatment control, FESS Sinu-Cleanse Hypertonic increased paracellular passage of FITC-dextrans by $96.6 \%, 108$ $\%, 140.2 \%, 594.4 \%$ and $837.9 \%$ after $30 \mathrm{~min}, 1 \mathrm{~h}, 2 \mathrm{~h}, 4 \mathrm{~h}$ and 6 h, respectively (Figure 6). 


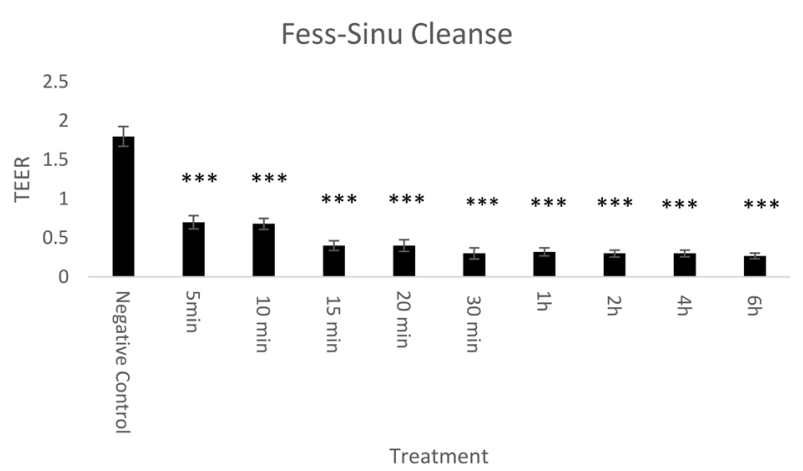

Figure 5. Trans-Epithelial Electrical Resistance (TEER) of HNEC monolayers derived from controls over time. TEER was measured at different time-points (5 min, $10 \mathrm{~min}, 15 \mathrm{~min}, 20 \mathrm{~min}, 30 \mathrm{~min}, 1 \mathrm{~h}, 2 \mathrm{~h}, 4 \mathrm{~h}$ and 6 h) in non-CRS control patients relative to untreated control. Time 0 is negative control (untreated), representing the TEER values $(\Omega / \mathrm{cm} 2)$ of the cells prior to application of the treatments. The values are shown as mean \pm SEM for $n=3 .{ }^{* * *}=p<0.001$, ANOVA, followed by Tukey HSD post hoc test.
FESS-Sinu Cleanse

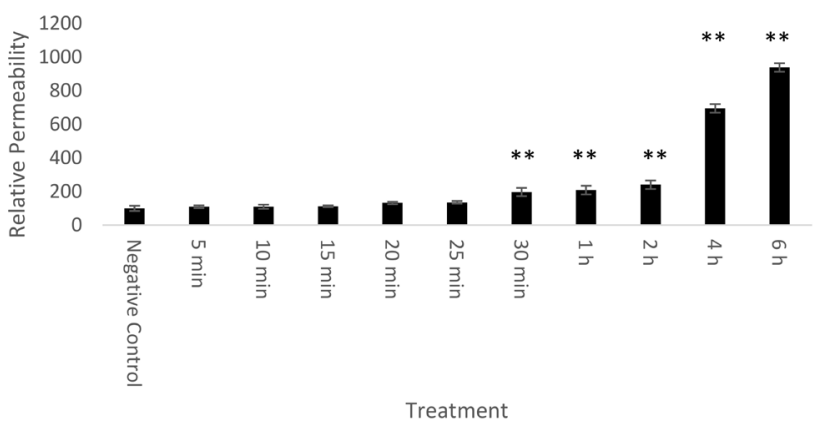

Figure 6. Paracellular passage of FITC-Dextrans over time. The passage of FITC-dextrans was measured over time and relative to no treatment control in HNEC monolayers from non-CRS control patients. The values are shown as means \pm SEM for $n=3$. Treatments significantly different from the untreated control at $p<0.01$ are presented as ${ }^{* *}$. ANOVA, followed by Tukey HSD post hoc test.
Effect of topical treatments on Lactate Dehydrogenase release from HNEC monolayers derived from controls over time.

The effect of FESS Sinu-Cleanse Hypertonic on LDH release from HNEC monolayers from control patients at different time points up to $6 \mathrm{~h}$. There was a statistically significant increase in LDH release after 6 hours incubation with FESS Sinu-Cleanse Hypertonic $(p=0.035)$ (Figure 7).

Effect of a brief exposure to FESS Sinu-Cleanse Hypertonic on HNEC monolayers from non-CRS control patients. To determine the effect of a brief exposure of HNEC-ALI cultures to FESS Sinu-Cleanse Hypertonic, the solution was applied to the cells for 5 minutes followed by removal of the solution, washing with PBS and application of fresh media. After $24 \mathrm{~h}$ incubation, TEER, cell layer permeability and cell viability was measured. Application of FESS Sinu-cleanse significantly increased TEER values $(p<0.05)$, increased paracellular permeability $(p<0.01)$, and reduced the cell viability $(p<0.01)$ in HNEC-ALI monolayers.

\section{Discussion}

Saline irrigations are the most commonly used topical treatments for the medical management of CRS. Saline preparations vary from homemade solutions to different commercial overthe-counter formulations. The salt content within saline irrigation solutions varies from $0.9 \% \mathrm{NaCl}$ (isotonic saline solutions) to $3 \% \mathrm{NaCl}$ (hypertonic saline solutions) and it is generally agreed that regular application has beneficial effects on patients' CRS signs and symptoms ${ }^{(19)}$. However, information on their safety is sparse and their effect on mucosal barrier structure and function is unknown.

Apart from nasal burning and irritation, hypertonic saline improves sinus-related quality of life and decreases symptoms of sinusitis ${ }^{(20)}$. Our results indicate that hypertonic saline was not toxic and did not have detrimental effects on epithelial barrier function. Rather, application of the treatment appeared to significantly enhance the TEER of human nasal epithelial cell monolayers derived from CRS patients 24 hours after application. Hypertonic saline has also been shown to improve intestinal mucosal barrier function in association with decreased lung permeability after trauma-hemorrhagic shock which may limit the systemic immuno-inflammatory response to hemorrhagic shock (21). Interestingly, application of hypertonic saline to control HNEC monolayers did not affect TEER values. The significance of these findings is unclear but could indicate that hypertonic solutions might strengthen mucosal barrier function only in inflammatory conditions such as in CRS patients. Also, the notion that the observed effect is donor-dependent, indicates differences in the physiology of CRS and control HNECs, even after multiple in vitro cell divisions took place. Isotonic saline (FLO Sinus Care) is another common irrigation solution that has shown beneficial effects for CRS patients. Some studies showed isotonic saline provided a greater improvement in mucociliary clearance and nasal airway patency compared with normal saline ${ }^{(22,23)}$. In this study, FLO Sinus Care isotonic saline had no effect on either TEER or paracellular permeability, led to no alterations in the localization of ZO-1 and was non-toxic. Topical corticosteroids including fluticasone and Budesonide have shown to be effective for the treatment of CRS and are often considered a first-line treatment 
Fess-Sinu Cleanse

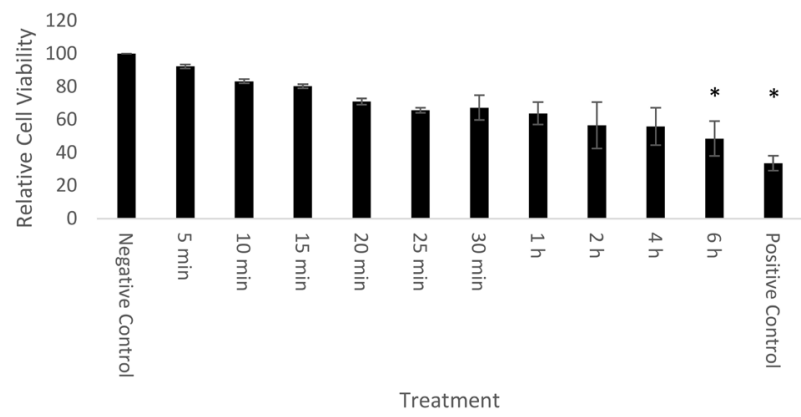

Figure 7. Relative cell viability measured by Lactate Dehydrogenase (LDH) assay of HNEC monolayers over time. Relative Viability as determined by the LDH assay at different time-points $(5 \mathrm{~min}, 10 \mathrm{~min}, 15 \mathrm{~min}$, $20 \mathrm{~min}, 30 \mathrm{~min}, 1 \mathrm{~h}, 2 \mathrm{~h}, 4 \mathrm{~h}$ and $6 \mathrm{~h}$ ) after application of FESS SinuCleanse Hypertonic on HNEC monolayers from non-CRS control patients. Cell viability was calculated relative to the untreated cells as negative control. Sodium Dodecyl Sulphate (SDS) in B-ALI complete medium was used as a positive control. The values are shown as means $\pm S E M, n=3$, $p=0.035$.

option for CRS with nasal polyps ${ }^{(24,25)}$. Our findings showed that Budesonide did not compromise the structure and function of the epithelial barrier, and did not cause cellular toxicity. In fact, Budesonide significantly increased TEER in CRS patients derived HNEC monolayers similarly to the effect seen with hypertonic saline solution. Glucocorticoids have been shown to enhance barrier function of lung epithelia via modulation of claudin 8 (cldn8) dependent recruitment of occludin at the tight junctions (26). Also in different in vitro models of blood-brain barrier function, corticosteroids were shown to enhance barrier function by a corticosteroid-receptor mediated effect on the expression of different tight junction proteins, including Occludin, Claudin-5 and ZO-1 ${ }^{(27,28)}$. Also fluticasone propionate has been shown to restore the barrier function in the nasal epithelium of patients with allergic rhinitis ${ }^{(29)}$.

Our results showed that application of FESS Sinu-Cleanse Hypertonic caused a significant reduction in TEER values followed by an increase in paracellular permeability of FITC-Dextrans. These results indicate that FESS Sinu-Cleanse Hypertonic negatively affects the mucosal barrier function of the sino-nasal epithelium in vitro. The mechanism of action of this effect and whether a specific ingredient in FESS Sinu-Cleanse Hypertonic might be responsible is unknown. FESS Sinu-Cleanse Hypertonic contains $20 \mathrm{mg} / \mathrm{g}$ Sodium Chloride and an undisclosed amount of Sodium bicarbonate and Xylitol and is mainly used during postoperative nasal care. The mean $\mathrm{pH}$ and osmolarity have been shown to be respectively 8.05 and $778 \mathrm{mmol} / \mathrm{l}$, making this solution hyper-osmolar ${ }^{(29)}$. FESS Sinu-Cleanse has been estimated to have a high ionic strength of $>500 \mathrm{mM}$ and compromised the
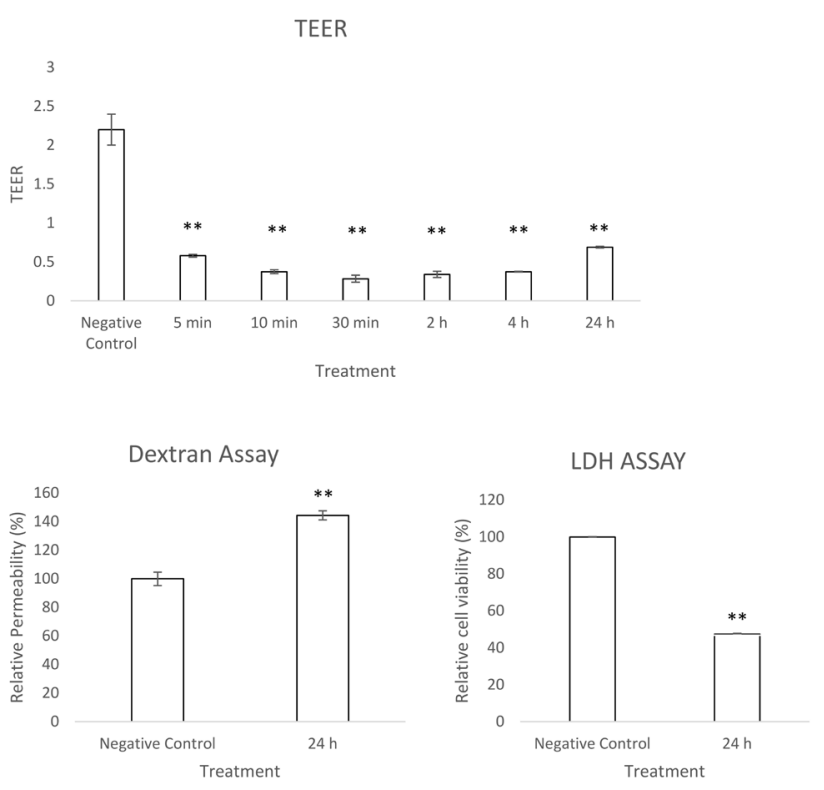

Figure 8. Effect of FESS Sinu-Cleanse Hypertonic on Trans-Epithelial Electrical Resistance, Paracellular permeability and cell viability of HNEC monolayers derived from control patients. A 5 minute application of FESS Sinu-Cleanse Hypertonic, followed by washing, followed by wash with PBS and application of medium, was followed by TEER measurements at different time points (5min, $10 \mathrm{~min}, 30 \mathrm{~min}, 2 \mathrm{~h}$, $4 \mathrm{~h}$ and 24h) (A); Paracellular permeability of FITC-dextrans measured after 24h (B); cell viability measured using an Lactate Dehydrogenase (LDH) assay after 24 $h(C)$. The values are shown as means \pm SEM, $n=3,{ }^{* * *}=p<0.001,{ }^{* *}=$ $p<0.01$. The FITC-dextrans and LDH assay statistical analysis were carried out using t-tests and the TEER analysis was performed using ANOVA, followed by Tukey's HSD post hoc test.

fungicidal activity of lysozyme, an innate immune peptide with bactericidal and fungicidal activity. This effect was not specific for FESS Sinu-Cleanse however as different (commercial) nasal irrigation solutions including nasal saline $(0.9 \% \mathrm{NaCl})$ could inhibit the fungicidal effects of lysozyme in vitro ${ }^{(30)}$. It is well known that the ionic strength of solutions applied to epithelial cell monolayers can influence TEER readings and it could be that the acute observed effect of reduced TEER values is at least in part dependent on the high ionic strength of FESS Sinu-Cleanse. Another ingredient of FESS Sinu-Cleanse is Xylitol, a naturally occurring penta-hydroxy sugar-alcohol that is present in a wide variety of fruits and vegetables. Xylitol is a safe and well tolerated compound, has established anti-bacterial and anti-biofilm activities and in a recent randomized, double-blinded, controlled crossover pilot study, it was shown that Xylitol in water was well-tolerated for sinonasal irrigation and resulted in greater improvement of symptoms of CRS compared to saline irrigation (31). Further experimentation would be needed to determine the molecular mechanisms and responsible factors of mucosal bar- 
rier dysfunction after application of FESS Sinu-Cleanse Hypertonic. Regardless, our investigations used an in vitro assay and it is premature to draw conclusions for the in vivo situation. Namely, whilst in our in vitro assay, the solutions are in direct contact with the HNEC monolayers for up to 24 hours, nasal irrigation solutions are typically flushed through the nose and therefore only have brief direct encounters with the mucosal surface in vivo. FESS Sinu-Cleanse Hypertonic is a buffered hypertonic solution and is specifically promoted to clear the nasal and sinus cavities of dried blood and mucus post sinus surgery and it may well be that the solution is well suited for this specific purpose. Buffered hypertonic solutions are indeed known to accelerate mucociliary and mucus clearance and can improve the lung function of cystic fibrosis patients ${ }^{(32,33)}$. Further comprehensive safety and efficacy evaluations and controlled clinical studies are needed to determine the effects of different commercial products on mucus clearance and mucosal barrier function in vivo.

\section{Acknowledgements}

This study was supported by a Conjoint Grant from The Garnett Passe and Rodney Williams Memorial Foundation to SV

\section{Authorship contribution}

Writing: MR; Design of the study: SV; Interpretation: MR, SV; Data collection: MR, AR; Statistical Analysis: JS.

\section{Conflict of interest}

The authors have no conflicting financial interests that are relevant to this work.

\section{References}

1. Blackwell D, Lucas J, Clarke T. Summary health statistics for U.S. adults: national health interview survey, 2012. Vital Health Stat 10. 2014;260:1-161

2. Rybak L. Medical treatment of chronic sinusitis in the immunocompetent and immunosuppressed patient: a review. Otolaryngol Head Neck Surg. 1982;90(5):534-9.

3. Soyka MB, Wawrzyniak P, Eiwegger $T$, Holzmann D, Treis A, Wanke K, et al. Defective epithelial barrier in chronic rhinosinusitis: the regulation of tight junctions by IFN- $\gamma$ and IL-4. J Allergy Clin Immunol. 2012;130(5):1087-96. e10

4. Wise SK, Laury AM, Katz EH, Den Beste KA, Parkos CA, Nusrat A, editors. Interleukin-4 and interleukin-13 compromise the sinonasal epithelial barrier and perturb intercelIular junction protein expression. Int Forum Allergy Rhinol. 2014 May;4(5):361-70.

5. Edwards A, Massey R. How does Staphylococcus aureus escape the bloodstream? Trends Microbiol. 2011;19(4):184-90.

6. Malik Z, Roscioli E, Murphy J, Ou J, Bassiouni A, Wormald PJ, et al., editors. Staphylococcus aureus impairs the airway epithelial barrier in vitro. Int Forum Allergy Rhinol. 2015 Jun;5(6):551-6.

7. Ramezanpour M, Moraitis M, Smith J, Wormald PJ, Vreugde S. Th17 Cytokines Disrupt the Airway Mucosal Barrier in Chronic Rhinosinusitis. Mediators Inflamm. 2016;2016:9798206.

8. Rogers G, Den BK, Parkos C, Nusrat A, Delgaudio J, Wise S. Epithelial tight junction alterations in nasal polyposis. Int Forum Allergy Rhinol. 2011;1(1).

9. Sinn P, Burnight E, Hickey M, Blissard G, McCray PJ. Persistent gene expression in mouse nasal epithelia following feline immunodeficiency virus-based vector gene transfer. J Virol. 2005;79(20):12818-2

10. Meltzer EO, Hamilos DL, Hadley JA,
Lanza DC, Marple BF, Nicklas RA, et al. Rhinosinusitis: establishing definitions for clinical research and patient care. J Allergy Clin Immunol. 2004 Dec;114(6 Suppl):155212.

11. Kern EB, Sherris D, Stergiou AM, Katz LM Rosenblatt LC, Ponikau J. Diagnosis and treatment of chronic rhinosinusitis: focus on intranasal Amphotericin B. Ther Clin Risk Manag. 2007 Jun;3(2):319-25.

12. Snidvongs K, Kalish L, Sacks R, Craig J Harvey R. Topical steroid for chronic rhinosinusitis without polyps. Cochrane Database Syst Rev. 2011;10(8).

13. Lal D, Hwang P. Oral corticosteroid therapy in chronic rhinosinusitis without polyposis: a systematic review. Int Forum Allergy Rhinol. 2011;1(2):136-43.

14. Slavin RG, Spector SL, Bernstein IL, Workgroup SU, Kaliner MA, Kennedy DW, et al. The diagnosis and management of sinusitis: a practice parameter update. J Allergy Clin Immunol. 2005;1 16(6):S13-S47.

15. Sastre J, Mosges R. Local and systemic safety of intranasal corticosteroids. J Investig Allergol Clin Immunol. 2012;22(1):1-12.

16. Sastre J, Mosges R. Local and systemic safety of intranasal corticosteroids. J Investig Allergol Clin Immunol. 2012;22(1):1-12.

17. Heatley D, McConnell K, Kille T, Leverson G. Nasal irrigation for the alleviation of sinonasal symptoms. Otolaryngol Head Neck Surg. 2001;25(1):44-8

18. Kumar RA, Viswanatha B, Krishnamurthy $N$, Jayanna N, Shetty DR. Efficacy of Hypertonic Saline and Normal Saline in the Treatment of Chronic Sinusitis. 2013. Int J Otolaryngol Head Neck Surg. 2013, 2, 90-96

19. Harvey R, Hannan SA, Badia L, Scadding G. Nasal saline irrigations for the symptoms of chronic rhinosinusitis. Cochrane Database Syst Rev. 2016 Apr 25:4:CD006394. .

20. Rabago D, Zgierska A, Mundt M, Barrett B, Bobula J, Maberry R. Efficacy of daily hypertonic saline nasal irrigation among patients with sinusitis: a randomized controlled trial. J Fam Pract. 2002;51(12):1049-55.

21. Shi HP, Deitch EA, Lu Q, Hauser CJ. Hypertonic saline improves intestinal mucosa barrier function and lung injury after trauma-hemorrhagic shock. Shock. 2002;17(6):496-501.

22. Hauptman G, Ryan M. The effect of saline solutions on nasal patency and mucociliary clearance in rhinosinusitis patients. Otolaryngol Head Neck Surg. 2007;137(5):815-21.

23. Keojampa B, Nguyen M, MW. R. Effects of buffered saline solution on nasal mucociliary clearance and nasal airway patency. Otolaryngol Head Neck Surg. 2004;131(5):679-82.

24. Joe S, Thambi R, Huang J. A systematic review of the use of intranasal steroids in the treatment of chronic rhinosinusitis. Otolaryngol Head Neck Surg. 2008;139(3):340-7.

25. Badia L, Lund V. Topical corticosteroids in nasal polyposis. Drugs. 2001;61(5):573-8.

26. Kielgast $F$, Schmidt $H$, Braubach $P$, Winkelmann VE, Thompson KE, Frick M, et al. Glucocorticoids Regulate Tight Junction Permeability of Lung Epithelia by Modulating Claudin 8. Am J Respir Cell Mol Biol. 2016 May;54(5):707-17.

27. Hue CD, Cho FS, Cao S, Cameron R, Meaney DF, Morrison B. Dexamethasone potentiates in vitro blood-brain barrier recovery after primary blast injury by glucocorticoid receptor-mediated upregulation of ZO-1 tight junction protein. J Cereb Blood Flow Metab. 2015 Jul;35(7):1191-8.

28. Förster C, Burek M, Romero IA, Weksler B, Couraud PO, Drenckhahn D. Differential effects of hydrocortisone and TNFa on tight junction proteins in an in vitro model of the human blood-brain barrier. J Physiol. 2008 Apr 1;586(7):1937-49.

29. Lilic N, Waldvogel-Thurlow S, Douglas R. Physical characteristics of commercial 
and home-made nasal lavage solutions. Laryngol Otol. 2014 Jan;128 Suppl 1:S40-3.

30. Woods CM, Hooper DN, Ooi EH, Tan L-W, Carney AS. Fungicidal activity of lysozyme is inhibited in vitro by commercial sinus irrigation solutions. Am J Rhinol Allergy. 2012 Jul-Aug;26(4):298-301.

31. Weissman JD, Fernandez F, Hwang PH. Xylitol nasal irrigation in the management of chronic rhinosinusitis: a pilot study. Laryngoscope. 2011;121(11):2468-72.

32. Donaldson SH, Bennett WD, Zeman KL, Knowles MR, Tarran R, Boucher RC. Mucus clearance and lung function in cystic fibrosis with hypertonic saline. N Engl J Med. 2006 Jan 19;354(3):241-50.

33. Talbot AR, Herr TM, Parsons DS. Mucociliary clearance and buffered hypertonic saline solution. Laryngoscope. 1997;107(4):500-3.
Dr. Sarah Vreugde

Department of Otorhinolaryngology

Head and Neck Surgery

The Queen Elizabeth Hospital

28 Woodville Rd

Woodville South,

South Australia 5011

Australia

Phone: +61-8-8222 7158

Fax: $+61-8-82227419$

E-mail:

sarah.vreugde@adelaide.edu.au 